\title{
Aproveitamento dos efluentes domésticos para geração de energia em condomínios residenciais de baixa renda
}

\section{Domestic wastewater utilization for Power Generation in low-income Residential Condominiums}

Data de entrada: 09/06/2016

Data de aprovação: 10/11/2017

\section{Resumo}

O estudo busca avaliar o potencial energético da estação de tratamento de esgoto de condomínios residenciais de baixa renda, realizando as seguintes estimativas: produção de biogás, produção de energia elétrica, necessidade energética do condomínio e avaliação econômica. Foram contabilizadas as perdas de metano durante o processo de tratamento anaeróbico. A produção média estimada de biogás foi de $60 \mathrm{~m}^{3} / \mathrm{d}$, resultando em uma possível disponibilidade de energia elétrica de 189,45 kWh/d, capaz de fornecer a iluminação dos postes e da área de lazer do condomínio. Além da possibilidade de atender à população local, pode-se ganhar créditos revertendo a energia elétrica gerada na rede de distribuição por meio do sistema de compensação de energia elétrica da Aneel. A utilização do biogás como geração de energia elétrica é importante para o meio ambiente, pois evita que seja lançado gás metano na atmosfera e reduz a captação dos recursos hídricos naturais com a geração de energia. Palavras-chave: Potencial energético. Biogás. Sustentabilidade.

\section{Abstract}

The study aims to assess the energy potential of the sewage treatment plant, low-income residential condominiums by performing the following estimates: biogas production, electricity production, energy needs of the community and economic evaluation. They were accounting for losses of methane during the anaerobic treatment process. The average estimated production of biogas was $60 \mathrm{~m}^{3} / \mathrm{d}$, resulting in a possible availability of electricity 189,45 kWh/d capable of providing illumination of streetlights and condominium recreation area. Besides the possibility to meet the local people, you can earn credits by reversing the electric power generated in the distribution network through the power compensation system Aneel. The use of biogas as electricity generation is important for the environment because it prevents methane gas is released into the atmosphere and reduces the uptake of natural water resources to generate energy. Keywords: Potential energy. Biogas. Sustainability.

\footnotetext{
Mayara Beuttenmüller Castro de Menezes - Engenheira Civil pela Universidade Federal de Alagoas. Mestre em Recursos Hídricos e Saneamento pela Universidade Federal de Alagoas CTEC/ UFAL. Gerente de projetos da empresa Tecnologia em Projetos e Construções - TPC.

Karina Ribeiro Salomon - Engenheira Agrônoma pela Universidade Federal de Lavras. Mestre em Engenharia de Energia pela Universidade Federal de Itajubá. Doutora em Engenharia Mecânica pela Universidade Federal de Itajubá. Docente do Programa de Pós-graduação em Recursos Hídricos e Saneamento CTEC/UFAL.

"Endereço para correspondência: Rua Oscar Araújo, 19 E - Massagueira - Marechal Deodoro - AL - CEP: 57160-000. Telefone: (82) $3327-3009$. E-mail: Eng.mayaraatpcprojetos.com.br.
} 


\section{INTRODUÇÃO}

Nas últimas décadas, o crescimento populacional trouxe como consequência o aumento de resíduos, e por falta de infraestrutura estes são depositados em locais impróprios (rios, solo), causando desequilíbrio ecológico, transmissão de doenças e prejudicando o meio ambiente. Estima-se que a população alcance 8,5 bilhões em 2030, chegue a 9,7 bilhões até 2050 e passe os 11,2 bilhões em 2100 (WORLD POPULATION, 2015). Isso faz com que sejam tomadas medidas para reaproveitamento de resíduos de forma sustentável (POLONIO et al, 2015)

O reúso de água, em especial de efluentes domésticos tratados, é uma prática incorporada em alguns países. Israel recicla $80 \%$ do efluente doméstico gerado, e quase tudo é encaminhado para a agricultura. No Japão, $40 \%$ do volume de efluente urbano tratado é reutilizado por indústrias (JIMÉNEZ e ASANO, 2008; OSORIO (2013 apud LIBHABER e CROOK, 2012)). A disponibilidade de esgoto doméstico é constante, fator esse que viabiliza o reúso e proporciona vários benefícios, tais como: a redução da captação dos recursos hídricos naturais; a reciclagem de nutrientes na agricultura, a economia de insumos (adubos e fertilizantes, $p$. ex.) e previne a poluição com a redução do lançamento de efluentes em corpos hídricos (FLORENClO, et al., 2006).

Existe grande variabilidade de aproveitamento para efluentes domésticos tratados. As aplicações são muitas, por exemplo: recreação, agricultura, dessedentação de animais, processos industriais e outras aplicações (HELMER e HESPANHOL, 1997).

O princípio do tratamento sanitário em reatores anaeróbios é a digestão anaeróbia de compostos orgânicos, que gera dois produtos principais, sendo a maior parte composta pelo biogás (70 a 90\%) e, em menor proporção, pelo lodo excedente do sistema (5 a 15\%) (SPERLING, 1997).
A utilização do biogás como combustível para geração de energia elétrica não apenas aproveita de forma sustentável esse subproduto da disposição dos resíduos sólidos como também evita que o gás metano $\left(\mathrm{CH}_{4}\right)$ nele contido seja emitido para a atmosfera. Conforme Godoy Júnior et al. (2004) apud ARCADIS Tetraplan (2010), o $\mathrm{CH}_{4}$ presente no biogás de esgoto é cerca de 21 vezes mais danoso para o meio ambiente do que o $\mathrm{CO}_{2}$, e a queima do biogás na produção de energia gera emissões evitadas deste gás. Seria interessante o aproveitamento energético do biogás proveniente da quantidade de carbono, sendo assim, impedido de alcançar a atmosfera da Terra, conciliando a geração de energia elétrica renovável com a questão do saneamento ambiental.

A Resolução Normativa 687/15 da Aneel estabeleceu as condições gerais para o acesso de microgeração e minigeração distribuída aos sistemas de distribuição de energia elétrica, e também instituiu o método de compensação de energia elétrica. A criação dessa resolução foi um importante passo para o crescimento da geração distribuída no Brasil, e é possível compensar os créditos de energia gerado nos demais meses.

Ultimamente tem-se discutido bastante a questão energética. Sabe-se que os combustíveis fósseis, além de altamente poluentes, também são considerados recursos não-renováveis, e as reservas naturais não devem durar muito tempo. Assim, soluções energéticas limpas e renováveis têm sido alvo de muita pesquisa. As tecnologias à base de fontes renováveis são atrativas não só devido às vantagens ambientais mas também sociais e econômicas.

O presente trabalho tem como objetivo avaliar o potencial energético do biogás de uma estação de tratamento de esgoto, em um condomínio residencial de baixa renda, e por meio dessa avaliação verificar a necessidade de demanda energética das áreas comuns do empreendimento e 
realizar a análise de viabilidade econômica da reutilização do biogás em forma de energia elétrica, para o empreendimento ou para a concessionaria elétrica local.

\section{OBJETIVOS}

Avaliar a produção de energia elétrica e a viabilidade econômica do potencial energético no biogás gerado a partir de ETE em um condomínio residencial de baixa renda.

\section{METODOLOGIA}

Maceió, cidade na qual está implantada o objeto de estudo, situa-se no litoral oriental do Nordeste brasileiro (Figura 1), encontra-se entre a latitude 9०33'18" Sul e longitude 3545'33" Oeste Datum SAD 69, às margens do Oceano Atlântico, e do complexo lagunar Mundaú - Manguaba. Tem como limites ao norte os municípios de Flexeiras, Paripueira e Messias; ao sul, Coqueiro Seco, Santa Luzia do Norte, Satuba e Marechal Deodoro; a oeste, Satuba, Rio Largo e Messias e a leste o Oceano Atlântico.

Figura 1- Local onde está inserido o objeto de estudo.

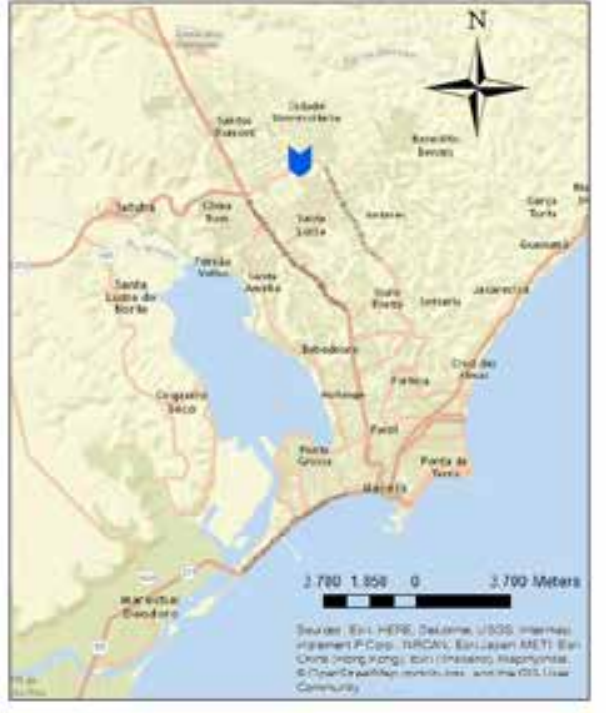

a)

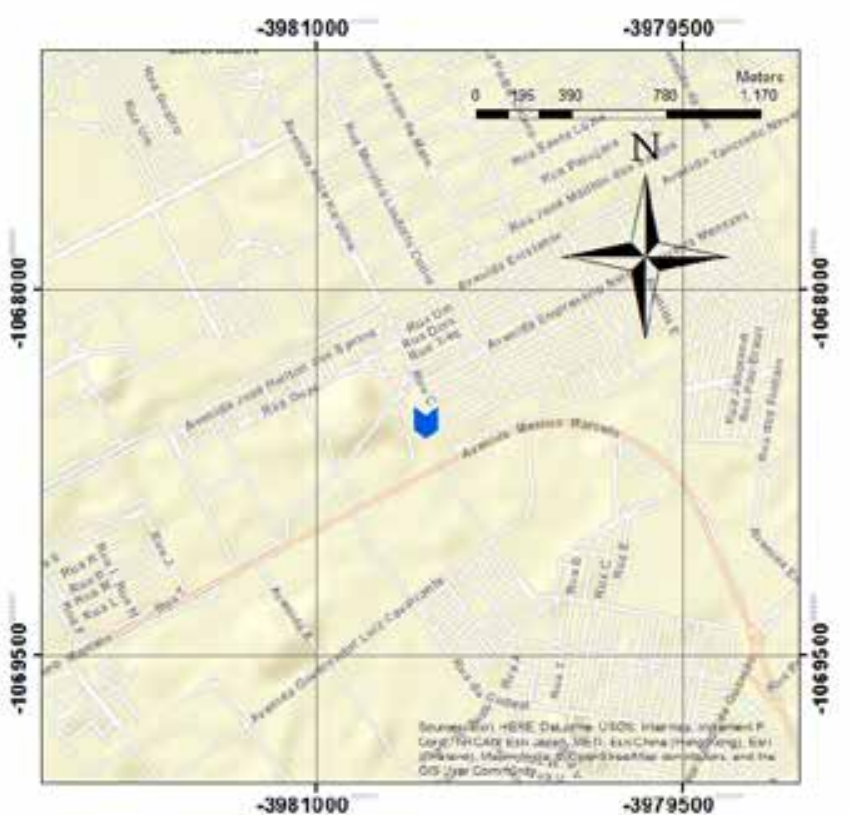

b)

Legenda: a) Cidade de Maceió - capital de Alagoas; b) Bairro Cidade Universitária com seus bairros adjacentes dentro da cidade de Maceió.

Fonte: Elaborado pelo autor, 2016

O empreendimento está localizado na Avenida Menino Marcelo, no bairro da Cidade Universitária, próximo ao Shopping Pátio Maceió, no município de Maceió/AL (Figura 1), possuindo um total de 528 unidades dividido em 6 torres e área de lazer comum as torres.

\subsection{Estimativa de Produção de Biogás}

A vazão per capita média de esgotos conforme Sperling (1997) é dada pela equação 1.

$$
\mathrm{Q}_{\text {méd }}=\frac{\mathrm{Q}_{\mathrm{pc}} \times \mathrm{r} \times \text { Pop }}{1000}
$$

(Equação 1) 
Onde:

$\mathrm{Q}_{\text {méd }}=$ Vazão média de esgoto em $\left(\mathrm{m}^{3} \mathrm{~d}^{-1}\right)$

$r=$ Coeficiente de retorno (esgoto /água)- recomenda $r=0,80$

$\mathrm{Q}_{\mathrm{pc}}=$ Consumo de água per capita $\left(\mathrm{l} \mathrm{hab}^{-1} \mathrm{~d}^{-1}\right)$

$\mathrm{Pop}=\mathrm{N}^{\circ}$ de habitantes

Nos cálculos de produção de metano foram adotadas as metodologias descritas por Lobato (2011), Zilotti (2012) e Bilotta (2014) conforme equações abaixo:

A estimativa da massa diária de DQO removida no sistema é dada pela equação 2

$\mathrm{R}_{\mathrm{DQO}}^{\mathrm{rem}}=\operatorname{Pop} \times \mathrm{QPC}_{\mathrm{DQO}} \times \frac{\mathrm{E}_{\mathrm{DQO}}}{100} \quad$ (Equação 2)

Onde:

$\mathrm{R}_{\mathrm{DQO}}^{\mathrm{rem}}=$ Carga diária de DQO removida no sistema $\left(\mathrm{kgDQO} \mathrm{dia}^{-1}\right)$

$\mathrm{QPC}_{\mathrm{DQO}}=$ Contribuição per capita de DQQ $\left(\mathrm{kg} \mathrm{hab}^{-1} \mathrm{dia}^{-1}\right)$

$\mathrm{E}_{\mathrm{DQO}}=$ Eficiência de remoção de DQQ (\%)

$\mathrm{Pop}=\mathrm{N}^{\circ}$ de habitantes

A estimativa da massa diária de DQO utilizada pela biomassa é dada pela equação 3 .

$\mathrm{R}_{\mathrm{DQO}}^{\text {lodo }}=\mathrm{R}_{\mathrm{DQO}}^{\text {rem }} \times \mathrm{Y} \times \mathrm{K}_{\text {sólidos }} \quad($ Equação 3)

Onde:

$\mathrm{R}_{\mathrm{DQO}}^{\text {lodo }}=$ Carga diária de DQO convertida em biomassa $\left(\mathrm{kgDQO}_{\text {lodo }} \mathrm{dia}^{-1}\right)$

$\mathrm{R}_{\mathrm{DQO}}^{r e m}=$ Carga diária de DQO removida no sistema $\left(\mathrm{kgDQO} \mathrm{dia}^{-1}\right)$

$\mathrm{Y}=$ Coeficiente de produção de sólidos no sistema (kgSTV/kgDQOrem)

$\mathrm{K}_{\text {sólidos }}=$ Fator de conversão de STV em DQQ (kgDQO $\left.{ }_{\text {lodo }} / \mathrm{kgSTV}\right)$
A estimativa da carga de sulfato convertida em sulfeto é dada pela equação 4 .

$\mathrm{R}_{\mathrm{SO}_{4}}^{\text {con }}=\mathrm{Q}_{\text {méd }} \times \mathrm{C}_{\mathrm{SO}_{4}} \times \frac{\mathrm{E}_{\mathrm{SO}_{4}}}{100} \quad$ (Equação 4)

Onde:

$\mathrm{R}_{\mathrm{SO}_{4}}^{\mathrm{con}}=$ Carga de $\mathrm{SO}_{4}$ convertida em sulfeto $\left(\mathrm{kgSO}_{4}\right.$ $\left.\operatorname{dia}^{-1}\right)$

Qméd = Vazão média de esgoto $\left(\mathrm{m}^{3} \mathrm{~d}^{-1}\right)$

$\mathrm{C}_{\mathrm{SO}_{4}}=$ Concentração média de $\mathrm{SO}_{4}$ no afluente $\left(\mathrm{kgSO}_{4} \mathrm{dia}^{-1}\right)$

$\mathrm{E}_{\mathrm{SO}_{4}}=$ Eficiência de redução de sulfato (\%)

A estimativa da massa diária de DQO utilizada na redução de sulfato é dada pela equação 5 .

$\mathrm{R}_{\mathrm{DQO}}^{\mathrm{SO}_{4}}=\mathrm{R}_{\mathrm{SO}_{4}}^{\mathrm{con}} \times \mathrm{K}_{\mathrm{DQO}}^{\mathrm{SO}_{4}}$

(Equação 5)

Onde:

$\mathrm{R}_{\mathrm{DQO}}^{\mathrm{SO}_{4}}=\mathrm{DQO}$ utilizada pelas bactérias redutoras de sulfato $\left(\mathrm{kgDOO}_{\mathrm{SO} 4} \mathrm{dia}^{-1}\right)$

$\mathrm{K}_{\mathrm{DQO}}^{\mathrm{SO}_{4}}=\mathrm{DQO}$ consumida na redução de sulfato $\left(0,667 \mathrm{kgDQO} / \mathrm{kg}_{\text {sO4convertido }}\right)$ LOBATO (2011),

$\mathrm{R}_{\mathrm{SO}_{4}}^{\mathrm{con}}=$ Carga de $\mathrm{SO} 4$ convertida em sulfeto $\left(\mathrm{kgSO}_{4} \mathrm{dia}^{-1}\right)$

Por fim, a estimativa da massa diária de DQO convertida em metano é dada pela equação 6 .

$\mathrm{R}_{\mathrm{DQO}}^{\mathrm{CH}_{4}}=\mathrm{R}_{\mathrm{DQO}}^{\mathrm{rem}}-\mathrm{R}_{\mathrm{DQO}}^{\text {lodo }}-\mathrm{R}_{\mathrm{DQO}}^{\mathrm{SO}_{4}}$ (Equação 6)

Onde:

$\mathrm{R}_{\mathrm{DQO}}^{\mathrm{CH}_{4}}=$ Carga diária de DQO convertida em metano $\left(\mathrm{kgDQO}_{\mathrm{CH} 4} \mathrm{dia}^{-1}\right)$

$\mathrm{R}_{\mathrm{DQO}}^{\mathrm{rem}}=$ Carga diária de DQO removida no sistema $\left(\mathrm{kgDQOO} \mathrm{dia}{ }^{-1}\right)$

$\mathrm{R}_{\mathrm{DQO}}^{\text {lodo }}=$ Carga diária de DQO convertida em biomassa $\left(\mathrm{kgDQO}_{\text {lodo }} \mathrm{dia}^{-1}\right)$ 
$\mathrm{R}_{\mathrm{DQO}}^{\mathrm{SO}_{4}}=\mathrm{DQO}$ consumida na redução de sulfato $\left(0,667 \mathrm{kgDQO} / \mathrm{kg}_{\text {SO4convertido }}\right)$

A estimativa da quantidade diária de metano produzida é dada pela equação 7 e será utilizada para obtenção da estimativa real de metano mostrado a seguir.

$\mathrm{Q}_{\mathrm{CH}_{4}}=\frac{\mathrm{R}_{\mathrm{DQO}}^{\mathrm{CH}_{4}} \times \mathrm{R} \times(273+\mathrm{T})}{\mathrm{P} \times \mathrm{K}_{\mathrm{DQO}}} \quad$ (Equação 7)

Onde:

$\mathrm{QCH}_{4}=$ Produção volumétrica teórica de metano $\left(\mathrm{kgDOOCH}_{4} \mathrm{dia}^{-1}\right)$

$\mathrm{R}_{\mathrm{DQO}}^{\mathrm{CH}_{4}}=$ Carga diária de DQO convertida em metano $\left(\mathrm{kgDQO}_{\mathrm{CH} 4} \mathrm{dia}^{-1}\right)$

$\mathrm{R}=$ Constante dos gases $\left(8,314 \mathrm{~J} \mathrm{~mol}^{-1} \mathrm{~K}^{-1}\right)$

$\mathrm{T}=$ Temperatura operacional da $\operatorname{ETE}\left({ }^{\circ} \mathrm{C}\right)$

$\mathrm{P}=$ Pressão atmosférica $(1 \mathrm{~atm}=101325 \mathrm{~Pa})$

$\mathrm{K}_{\mathrm{DQO}}=\mathrm{DQO}$ correspondente a um mol de $\mathrm{CH}_{4}$ $\left(0,064 \mathrm{kgDOOmol}^{-1}\right)$

Para o cálculo da estimativa real de metano é necessário calcular as possíveis perdas na fase gasosa como: gás residual e como outras perdas e na fase líquida, conforme equações 8, 9 e 10.

$$
\begin{array}{ll}
\mathrm{Q}_{\mathrm{CH}_{4}}^{\mathrm{W}}=\mathrm{Q}_{\mathrm{CH}_{4}} \times \mathrm{p}_{w} & \text { (Equação 8) } \\
\mathrm{Q}_{\mathrm{CH}_{4}}^{\mathrm{O}}=\mathrm{Q}_{\mathrm{CH}_{4}} \times \mathrm{p}_{O} & \text { (Equação 9) }
\end{array}
$$$$
\mathrm{Q}_{\mathrm{CH}_{4}}^{L}=\mathrm{Q}_{m e ́ d} \times \mathrm{p}_{L} \times \mathrm{f}_{\mathrm{CH}_{4}} \times \frac{\mathrm{R} \times(273+\mathrm{T})}{\mathrm{P} \times \mathrm{K}_{\mathrm{DQO}}}
$$$$
\text { (Equação 10) }
$$

Onde:

$\mathrm{QCH}_{4}=$ Produção volumétrica teórica de metano $\left(\mathrm{kgDOOCH} \mathrm{dia}^{-1}\right)$
$\mathrm{Q}_{\mathrm{CH}_{4}}^{\mathrm{W}}=$ Perda de metano na fase gasosa, com o gás residual $\left(\mathrm{m}^{3} \mathrm{dia}^{-1}\right)$

$\mathrm{Q}_{\mathrm{CH}_{4}}^{\mathrm{O}}=$ Outras perdas de metano na fase gasosa $\left(m^{3} \mathrm{dia}^{-1}\right)$

$\mathrm{Q}_{\mathrm{CH}_{4}}^{L}=$ Perda de metano na fase líquida, dissolvido no efluente $\left(\mathrm{m}^{3} \mathrm{dia}^{-1}\right)$

$\mathrm{p}_{w}=$ Percentual de perda de metano na fase gasosa, com o gás residual (\%)

$\mathrm{p}_{O}=$ Percentual de outras perdas de metano na fase gasosa (\%)

$\mathrm{p}_{L}=$ Perda de metano na fase líquida, dissolvido no efluente $\left(\mathrm{kg} \mathrm{m}^{-3}\right)$

$\mathrm{f}_{\mathrm{CH}_{4}}=$ Fator de conversão de massa de metano em massa de DQO - coeficiente estequiométrico $\left(4 \mathrm{kgDOQO} / \mathrm{kgCH}_{4}\right)$

$\mathrm{R}=$ Constante dos gases $\left(8,314 \mathrm{~J} \mathrm{~mol}^{-1} \mathrm{~K}^{-1}\right)$

$\mathrm{T}=$ Temperatura operacional da $\operatorname{ETE}\left({ }^{\circ} \mathrm{C}\right)$

$\mathrm{P}=$ Pressão atmosférica $(1 \mathrm{~atm}=101325 \mathrm{~Pa})$

$\mathrm{K}_{\mathrm{DQO}}=\mathrm{DQO}$ correspondente a um mol de $\mathrm{CH}_{4}$ $\left(0,064 \mathrm{kgDQOmol}^{-1}\right)$

Agora, para obter a produção real de metano disponível para recuperação de energia basta retirar as perdas obtidas nas equações 8,9 e 10 da estimativa de produção diária obtida na equação 7 .

Finalmente pode-se realizar o cálculo da produção de biogás capturado por meio da equação 11 .

$\mathrm{Q}_{\text {biogás }}=\frac{\mathrm{Q}_{\mathrm{CH}_{4}}^{\text {real }}}{\mathrm{C}_{\mathrm{CH}_{4}}} \times 100$

(Equação 11)

Onde:

$\mathrm{Q}_{\text {biogás }}=$ Produção de biogás capturado $\left(\mathrm{m}^{3} \mathrm{dia}^{-1}\right)$ $\mathrm{Q}_{\mathrm{CH}_{4}}^{\mathrm{real}}=$ Produção real de metano disponível para recuperação de energia $\left(\mathrm{m}^{3} \mathrm{dia}^{-1}\right)$

$\mathrm{C}_{\mathrm{CH}_{4}}=$ Concentração de metano no biogás (\%) 


\subsection{Conversão Energética do Biogás}

Segundo Zilotti (2012), para transformar a energia do biogás em $\mathrm{kWh} / \mathrm{m}^{3}$ foi empregada a equação 12, que o transforma diretamente em energia por metro cúbico de biogás.

$\mathrm{PCl}_{\mathrm{d}}=\mathrm{PE} \times \mathrm{PCl} \times \mathrm{K}$

(Equação 12)

Onde:

$\mathrm{PCl}_{\mathrm{d}}=$ Potencial Calorífico Inferior disponível em $\mathrm{kWh} / \mathrm{m}^{3}$;

$\mathrm{PE}=$ Peso Específico em $\mathrm{kg} / \mathrm{Nm}^{3}$;

$\mathrm{PCl}=$ Potencial calorífico inferior em $\mathrm{kcal} / \mathrm{kg}$;

$K=4,19 \mathrm{kWh} / 3600$. Constante de conversão entre $\mathrm{kcal} \longrightarrow \mathrm{kJoules} \longrightarrow \mathrm{kwh}$.

Para cálculo da potência elétrica efetiva foi utilizado o valor de eficiência global de 35\% para a conversão nas máquinas térmicas, com grupos geradores (motores ciclo Otto), e sistema de cogeração de 75 \% conforme Lobato (2011). Então, pela equação 13 , determinou-se a potência elétrica final a ser disponibilizada pelos geradores.

$\mathrm{P}=\mathrm{Q}_{\mathrm{CH}_{4}}^{\text {real }} \times \mathrm{PCI}_{\mathrm{d}} \times \mathrm{n}$

(Equação 13)

Onde:

$\mathrm{P}=$ Potência Elétrica Disponibilizada pelo metano, $\mathrm{kWh} / \mathrm{d}$

$\mathrm{Q}_{\mathrm{CH}_{4}}^{\mathrm{real}}=$ Produção real de metano disponível para recuperação de energia $\left(\mathrm{m}^{3} \mathrm{dia}^{-1}\right)$;

$\mathrm{PCl}_{\mathrm{d}}=$ Potencial Calorífico Inferior disponível em $\mathrm{kWh} / \mathrm{m}^{3}$;

n = Eficiência de conversão de máquinas.

\subsection{Necessidade Energética do Empreendimento}

Os critérios adotados pela Eletrobras com relação ao tipo de vias públicas classificam as ruas do Re- sidencial em ruas simples, com classe econômica considerada de classe $\mathrm{C}$.

Para a iluminação das ruas e da praça foi considerada a potência de 0,15 kVA por luminária, correspondente a 01 (uma) lâmpada de vapor de mercúrio de $125 \mathrm{~W}$, inclusa a perda no reator.

Para a iluminação da quadra de esportes foi considerada a potência de 0,29 kVA por projetor, correspondente a 01 (uma) lâmpada de vapor de mercúrio de $250 \mathrm{~W}$, inclusa a perda do reator.

Para o cálculo de queda de tensão na rede secundária foi utilizada a tensão nominal $220 / 380$ V, e o limite máximo aceitável de queda de tensão de $5 \%$.

Conforme NBR 5410/2005, na determinação das cargas de iluminação em cômodos ou dependências deve ser prevista uma carga mínima de 100 VA para os primeiros $6 \mathrm{~m}^{2}$, acrescida de $60 \mathrm{VA}$ para cada aumento de $4 \mathrm{~m}^{2}$ inteiros.

A demanda de energia da iluminação é obtida por meio da equação 14 (NTD - 001/2008) e equação 15.

№ de postes $=\frac{\text { Comprimento da rua em metros }}{35 \mathrm{~m}}$ (Equação 14)

Demanda de iluminação $=\mathrm{N}^{\circ}$ de postes $\times \mathrm{P}$ da luminária em kVA

(Equação 15)

\subsection{Resolução Aneel 687/15}

Na Resolução Normativa 687 da Aneel de 24 de novembro de 2015 (ANEEL, 2015) são definidas duas categorias em que se enquadram os pequenos produtores de energia elétrica: a primeira, de microgeração distribuída, contempla os produtores que possuem potência instalada de até $75 \mathrm{~kW}$ para cogeração qualificada; a segunda categoria, da minigeração distribuída, contempla os produtores que possuem potência instalada entre $75 \mathrm{~kW}$ a $3 \mathrm{MW}$ para fontes hídricas ou até $5 \mathrm{MW}$ para cogeração qualificada. Em ambos os casos, as fontes de energia devem ser provenien- 
tes de energia hidráulica, solar, eólica, biomassa ou para as demais fontes renováveis de energia elétrica, conectada na rede de distribuição por meio de instalações de unidades consumidoras, conforme regulamentação em questão (REN ANEEL 687, 2015).

O sistema de compensação de energia elétrica é o sistema no qual a energia ativa injetada por unidade consumidora com microgeração ou minigeração distribuída é cedida por meio de empréstimo gratuito à distribuidora local e posteriormente compensada com o consumo de energia elétrica ativa. A unidade consumidora passa a ter um crédito em quantidade de energia ativa a ser consumida por um prazo de 60 (sessenta) meses.

Os custos de eventuais melhorias ou reforços no sistema de distribuição em função exclusivamente da conexão de microgeração distribuída não devem fazer parte do cálculo da participação financeira do consumidor, sendo integralmente arcados pela distribuidora, exceto para o caso de geração compartilhada (ANEEL, 2015).

Para o caso de unidade consumidora com microgeração ou minigeração distribuída, o faturamento deve considerar a energia consumida, deduzidos o percentual de energia excedente alocado à essa unidade consumidora e eventual crédito de energia acumulado em ciclos de faturamentos anteriores, por posto tarifário, quando for o caso, sobre os quais deverão incidir todas as componentes da tarifa em $\mathrm{R} \$ / M W h$.

O excedente de energia é a diferença positiva entre a energia injetada e a consumida, exceto para o caso de empreendimentos de múltiplas unidades consumidoras, em que o excedente é igual à energia injetada.

Os créditos de energia ativa expiram em 60 meses após a data do faturamento e serão revertidos em prol da modicidade tarifária sem que o consumidor faça jus a qualquer forma de compensação após esse prazo.
A distribuidora é responsável técnica e financeiramente pelo sistema de medição para microgeração distribuída, de acordo com as especificações técnicas do PRODIST. Após a adequação do sistema de medição, a distribuidora será responsável por sua operação e manutenção, incluindo os custos de eventual substituição ou adequação.

\subsection{Avaliação Econômica}

Considerando condições ideais de operação e manutenção, a estação de tratamento pode injetar biogás no gerador durante todo o ano. Foi adotado um funcionamento em média de 10 horas por dia para o gerador; este valor foi adotado para os cálculos que consideram o tempo de operação.

Para implementar o sistema de geração de energia é necessário um investimento inicial para compra e instalação dos equipamentos. A equação 16 fornece a estimativa do investimento inicial.

$\|=C M+M O$

(Equação 16)

Onde:

II - Investimento inicial [R\$];

$\mathrm{CM}$ - Custos com materiais e equipamentos [R\$]; MO - Custos com mão de obra [R\$].

O método de depreciação utilizado foi o da depreciação linear, descrito por Bauer (2008), pelo qual a depreciação dos custos fixos ou variáveis é avaliada conforme mostra a equação 17 :

$\mathrm{D}=\frac{\mathrm{Ci}+\mathrm{Cf}}{\mathrm{Vu}}$

Em que,

D - Depreciação anual [R $\$$ ano-1];

$\mathrm{Ci}$ - Custos com materiais depreciáveis [R\$];

$\mathrm{CF}$-Valor final do ativo [R\$], e;

$\mathrm{Vu}$-Vida útil [ano]. 
Os juros sobre o capital investido foram determinados em relação ao capital médio durante a vida útil dos bens, a uma taxa de juros de 17,876\% ao ano (adotada a taxa de fevereiro de 2016 do BNDES), e segundo o método descrito por Bauer (2008); dado pela equação 18, os juros podem ser obtidos por:

$\mathrm{J}=\frac{\mathrm{Vi}+\mathrm{Vf}}{2} \mathrm{r}$

(Equação 18)

Para a qual;

J - Juros sobre capital investido [R $\$$ ano-1 $]$;

Vi - Valor total do investimento [R\$];

VF - Valor final do ativo [R\$];

$r$ - Taxa de juros [\% ano-1].

Os custos com a manutenção dos equipamentos consideram o intervalo de manutenção dos componentes e os valores cobrados pelos técnicos responsáveis. Portanto, o gasto anual pode ser obtido utilizando-se a equação 19:

$\mathrm{G}_{\mathrm{M}}=\frac{\mathrm{T}}{\mathrm{Im}} \mathrm{At}$

(Equação 19)

Para qual,

GM - Gastos com a manutenção [R\$ ano-1];

$\mathrm{T}$ - Tempo de operação [h ano-1];

IM - Intervalo de manutenção dos componentes [h], e AT - Assistência técnica [R\$].

Os custos anuais do sistema foram determinados considerando-se os custos fixos referentes à depreciação, aos juros sobre o capital investido e os custos variáveis referentes à manutenção e à operação.

A produção anual de biogás pode ser dada pela equação 20:

$\mathrm{P}_{\mathrm{AB}}=\mathrm{P}_{\mathrm{DB}} * \mathrm{~T}_{\mathrm{D}}$

(Equação 20)

Onde:

$\mathrm{P}_{\mathrm{AB}}$ - Produção anual de biogás $\left[\mathrm{m}^{3}\right.$ ano $\left.^{-1}\right]$;

$P_{D B}$ - Produção diária de biogás $\left[\mathrm{m}^{3} \mathrm{dia}^{-1}\right]$;

$\mathrm{T}_{\mathrm{D}}$ - Disponibilidade anual da planta [dias ano-1].
O consumo anual de biogás pelo gerador pode ser dado pela equação 21 :

$C_{A B}=C_{E B} * T_{D}$

Onde:

$\mathrm{C}_{\mathrm{AB}}$ - Consumo anual de biogás $\left[\mathrm{m}^{3}\right.$ ano-1];

$C_{E B}$ - Consumo específico de biogás pelo motor-gerador $\left[\mathrm{m}^{3} \mathrm{~h}^{-1}\right]$, e

$\mathrm{T}_{\mathrm{D}}$ - Disponibilidade anual da planta $[\mathrm{h}$ ano-1].

Considerando a quantidade de energia produzida de acordo com o consumo anual do grupo gerador e a tarifa de energia elétrica obtém-se o benefício que é interpretado como a redução do valor que é passado para distribuidora, e pode ser obtido pela equação 22:

$B_{G E E}=E_{E P} * T_{E E}$

(Equação 22)

Onde:

$\mathrm{B}_{\mathrm{GEE}}$ - Benefício com a geração de energia elétrica [R\$ ano-1];

$E_{E P}$ - Energia elétrica produzida [ $\mathrm{kWh}$ ano-1];

$\mathrm{T}_{\mathrm{EE}}$ - Tarifa de energia elétrica $\left[\mathrm{R} \$ \mathrm{kWh}^{-1}\right]$.

Os cálculos foram realizados com uma taxa de desconto da poupança, que possui taxa de juros $8,5 \%$ ao ano em média.

Com os custos e benefícios calculados foi possível determinar o fluxo de caixa do projeto e realizar a análise de viabilidade econômica por meio do Valor Presente Líquido (VPL), da Taxa Interna de Retorno (TIR) e pelo Período de Retorno do Capital (Payback).

O Valor Presente Líquido (VPL) é o retorno líquido atualizado gerado pelo projeto, que permite analisar a viabilidade econômica do projeto a longo prazo (Lapponi, 2000), e pode ser obtido pela equação 23

$$
V P L=-I I+\sum_{i=1}^{n} \frac{B i-C i}{(1+r)^{i}}
$$


A TIR pode ser calculada utilizando a equação 24

$T I R=\sum_{i=1}^{n} \frac{B i-C i}{(1+\propto)^{i}}=0$

(Equação 24)

Onde:

II -Investimento inicial [R\$];

$\mathrm{Bi}$ - Benefício do projeto, em unidades monetárias, no [ano i];

Ci - Custo do projeto, em unidades monetárias, no [ano i];

r - Taxa de desconto [\%a.a];

i - Contador de tempo, em [ano], e

$\mathrm{n}$ - Período de vida útil do investimento, em [ano].

$\propto$ : Taxa interna de retorno (TIR)

O objetivo do método do Período de Retorno do Capital é obter tempo de retorno do valor investido, utilizando a equação 25 :

$P R C=\frac{I I}{l u}$

(Equação 25)

Onde:

II -Investimento inicial [R\$];

lu - Lucro anual previsto [R $\$$ ano-1];

\subsection{Premissas Adotadas}

As principais variáveis necessárias para estimar a produção de metano e o potencial energético obtidos são as seguintes:

- População contribuinte: 2.544 hab;

- Contribuição per capita de esgoto: $96 \mathrm{~L} \mathrm{hab}^{-1} \mathrm{dia}^{-1}$

- Contribuição per capita de DQQO (Q $\left.{ }_{\mathrm{PCDQO}}\right): 0,10 \mathrm{~kg}$ DQO hab $^{-1} \mathrm{dia}^{-1}$ (SPERLING,1997)

- Eficiência de remoção de DQQO (EDQQO): 67,54 \% (FIBRATÉCNICA, 2011)

- Coeficiente de produção de sólidos (Y): 0,15 $\mathrm{kgDQO} \mathrm{Lodo}_{\text {Lo }} / \mathrm{kg}_{\text {DooRemov }}$ Lobato (2011) e Eller (2013)
- Fator de conversão de STV em DQQO (k sólidos): 1,42 Lobato (2011) e Eller (2013)

- Concentração de $\mathrm{SO}_{4}$ no afluente (COSO4): 0,039 $\mathrm{kgSO}_{4} / \mathrm{m}^{3}$ Eller (2013)

- Eficiência de redução de sulfato $\left(\mathrm{ESO}_{4}\right)$ : 61,52\% Eller (2013)

- DQQO consumida na redução de sulfato ( $\left.\mathrm{K}_{\mathrm{DQO}-\mathrm{SO} 4}\right)$ : 0,667 kgDQOSO $4 / \mathrm{kgSO}_{4}$ Eller (2013)

- Temperatura operacional do reator (T): $25^{\circ} \mathrm{C}$ (298,15 K) (BARBIRATO ET AL., 2002)

- Constante dos gases (R): 8,314 $\mathrm{J} \mathrm{mol}^{-1} \mathrm{~K}^{-1}$

- Pressão atmosférica (P): 101325 Pa

- DQO corresponde a $1 \mathrm{~mol}$ de metano (KDQO): 0,064 kgDQOCH $4 / \mathrm{mol}$ Lobato (2011) e Eller (2013)

- Percentual de $\mathrm{CH}_{4}$ no biogás $\left(\mathrm{C}_{\mathrm{CH} 4}\right): 65 \%$

- Perda de $\mathrm{CH}_{4}$ na fase gasosa $\left(\mathrm{p}_{w}\right): 5,0 \%$ Lobato (2011) e Eller (2013)

- Outras perdas de $\mathrm{CH}_{4}$ na fase gasosa $\left(\mathrm{p}_{O}\right): 5,0 \%$ Lobato (2011) e Eller (2013)

- Perda de $\mathrm{CH}_{4}$ dissolvido no efluente $\left(\mathrm{p}_{L}\right): 0,020$ $\mathrm{kg} / \mathrm{m}^{3}$ Lobato (2011) e Eller (2013)

- Fator de conversão teórico de DQO em $\mathrm{CH}_{4}\left(\mathrm{FCH}_{4}\right)$ : $4,0 \mathrm{kgDQO} / \mathrm{kgCH}_{4}$ Lobato (2011) e Eller (2013)

- Peso específico do $\mathrm{CH}_{4}$ (PE): $1,1518 \mathrm{~kg} / \mathrm{Nm}^{3}$ Lobato (2011) e Eller (2013)

- Poder calorífico inferior do $\mathrm{CH}_{4}(\mathrm{PCl}): 4.831,1$ $\mathrm{kcal} / \mathrm{kg}$ Lobato (2011) e Eller (2013)

- Valor de eficiência de 35\% para a conversão nas máquinas térmicas Lobato (2011)

- Valor de eficiência de 75\% para sistema de cogeração (considerando o aproveitamento da energia na forma de energia elétrica e calor); Lobato (2011)

- 1 mês = 30,4167 d (valor médio no período de 1 ano). 
As principais premissas e condições adotadas para avaliação econômica são as seguintes:

- A taxa de juros foi de 17,88 ao ano (BNDES, 2016);

- A depreciação é linear ao longo da vida;

- Não foi considerado o valor para o abrigo, pois o mesmo ficará na casa de máquinas da estação de tratamento existente.

- Para fins comparativos utilizou-se o valor da taxa de energia elétrica de $\mathrm{R} \$ 0,66$ kwh (ELETROBRAS, jan/2016);

- O valor do gerador de 18 c.v. : $\mathrm{R} \$ 16.500,00$; (AGROSHOP,2015)

\section{RESULTADO}

\subsection{Estimativa de Produção de Biogás}

Utilizando as premissas do item 4.6 e as equações do item 4.2 obtiveram-se os resultados conforme tabela 1:

Tabela 1 - Resultados calculados Estimativa de Produção de Biogás

\begin{tabular}{|c|c|c|}
\hline Variável & $\begin{array}{l}\text { Valor } \\
\text { obtido }\end{array}$ & Unidades \\
\hline $\begin{array}{l}\text { Estimativa da massa diária de DQQO } \\
\text { removida no sistema }\end{array}$ & 171,82 & kgDQO $\mathrm{dia}^{-1}$ \\
\hline $\begin{array}{c}\text { Estimativa da massa diária de DQQO } \\
\text { utilizada pela biomassa }\end{array}$ & 36,60 & kgDQQO lodo dia-1 \\
\hline $\begin{array}{l}\text { Estimativa da carga de sulfato } \\
\text { convertida em sulfeto }\end{array}$ & 0,06 & $\mathrm{kgSO}_{4} \mathrm{dia}^{-1}$ \\
\hline $\begin{array}{l}\text { Estimativa da massa diária de DQO } \\
\text { utilizada na redução de sulfato }\end{array}$ & 0,04 & $\mathrm{kgDQOSO}_{4} \mathrm{dia}^{-1}$ \\
\hline $\begin{array}{c}\text { Estimativa da massa diária de DQQO } \\
\text { convertida em metano }\end{array}$ & 135,13 & $\mathrm{kgDQO} \mathrm{CH}_{4} \mathrm{dia}^{-1}$ \\
\hline $\begin{array}{l}\text { Estimativa da quantidade diária de } \\
\text { metano produzida }\end{array}$ & 51,63 & $\mathrm{kgDQO} \mathrm{CH}_{4} \mathrm{dia}^{-1}$ \\
\hline $\begin{array}{l}\text { Estimativa das perdas de metano na } \\
\text { fase gasosa, como gás residual }\end{array}$ & 2,58 & $\mathrm{~m}^{3} \mathrm{dia}^{-1}$ \\
\hline $\begin{array}{c}\text { Estimativa de outras perdas de } \\
\text { metano na fase gasosa (vazamentos, } \\
\text { purgas de condensado, etc) }\end{array}$ & 2,58 & $\mathrm{~m}^{3} \mathrm{dia}^{-1}$ \\
\hline $\begin{array}{l}\text { Estimativa das perdas de metano na } \\
\text { fase líquida, dissolvido no efluente }\end{array}$ & 7,47 & $\mathrm{~m}^{3} \mathrm{dia}^{-1}$ \\
\hline $\begin{array}{l}\text { Estimativa da produção real de } \\
\text { metano }\end{array}$ & 39,00 & $\mathrm{~m}^{3} \mathrm{dia}^{-1}$ \\
\hline $\begin{array}{l}\text { Estimativa da produção de biogás } \\
\text { capturado }\end{array}$ & 60,00 & $\mathrm{~m}^{3} \mathrm{dia}^{-1}$ \\
\hline
\end{tabular}

Fonte: Elaborado pelo autor (2016)

\subsection{Conversão Energética do Biogás}

Utilizando os dados calculados na estimativa de produção do biogás capturado e produção real do metano obtiveram-se os resultados conforme tabela 2 :

Tabela 2 - Resultados calculadosEnergética do biogás

\begin{tabular}{|c|c|c|}
\hline \multicolumn{1}{|c|}{ Variável } & $\begin{array}{c}\text { Valor } \\
\text { obtido }\end{array}$ & Unidades \\
\hline $\begin{array}{c}\text { Poder calorífico inferior disponível } \\
\text { do } \mathrm{CH}_{4}\end{array}$ & 6,48 & $\mathrm{kWh} \mathrm{m}^{-3}$ \\
\hline Potência elétrica efetiva com $\mathrm{n}=0,35$ & 88,41 & $\mathrm{kWh} \mathrm{dia}^{-1}$ \\
\hline Potência elétrica efetiva com $\mathrm{n}=0,75$ & 189,45 & $\mathrm{kWh} \mathrm{dia}^{-1}$ \\
\hline
\end{tabular}

Fonte: Elaborado pelo autor2016

\subsection{Necessidade Energética do Empreendimento:}

Tabela 3- Resultados da demanda energética

\begin{tabular}{|c|c|c|}
\hline Ambiente & $\begin{array}{c}\text { Potência } \\
\text { demandada }\end{array}$ & Unidades \\
\hline Iluminação dos Postes (ruas internas) & 4,80 & kVA \\
\hline Área de lazer (iluminação) & 2,06 & kVA \\
\hline
\end{tabular}

\subsection{Avaliação Econômica}

Foram testados dois cenários: o primeiro com gerador pronto para utilizar biogás de fábrica produzindo energia para o empreendimento, e o segundo utilizando toda a energia produzida no primeiro cenário para devolver à rede de energia elétrica.

\subsubsection{Cenário 01}

Foi escolhido um gerador de $18 \mathrm{cv}$, com filtro incluso, que consome $4 \mathrm{~m}^{3}$ de biogás por hora e gera 9,5 kVA. O investimento inicial e os custos anuais de operação foram estabelecidos de acordo com informações fornecidas pela empresa de instalação e manutenção do gerador.

Na tabela 4 e 5 estão representados os valores de investimento, equipamento e material com seus juros e depreciações. 
Tabela 4 - Valores obtidos para os investimentos

\begin{tabular}{|c|c|}
\hline Investimento & Valor (R\$) \\
\hline Aquisição do grupo gerador & $\mathrm{R} \$ 7.500,00$ \\
\hline Instalações elétricas & $\mathrm{R} \$ 525,00$ \\
\hline Mão de obra para implantação & $\mathrm{R} \$ 225,00$ \\
\hline Total & $\mathrm{R} \$ 8.250,00$ \\
\hline
\end{tabular}

Tabela 5 - Depreciação dos bens depreciáveis

\begin{tabular}{|c|c|c|c|}
\hline $\begin{array}{c}\text { Equipamento / } \\
\text { material }\end{array}$ & $\begin{array}{c}\text { Valor inicial } \\
\text { (R\$) }\end{array}$ & $\begin{array}{c}\text { Vida útil } \\
\text { (ano) }\end{array}$ & $\begin{array}{c}\text { Depreciação } \\
\text { (R\$/ano) }\end{array}$ \\
\hline $\begin{array}{c}\text { Abrigo do conjunto } \\
\text { motor-gerador }^{1}\end{array}$ & $\mathrm{R} \$ 0,00$ & 20 & $\mathrm{R} \$ 0,00$ \\
\hline Instalações elétricas & $\mathrm{R} \$ 1.155,00$ & 20 & $\mathrm{R} \$ 57,75$ \\
\hline Grupo gerador & $\mathrm{R} \$ 16.500,00$ & 10 & $\mathrm{R} \$ 1.650,00$ \\
\hline Total & $\mathrm{R} \$ 17.655,00$ & & $\mathrm{R} \$ 1.707,75$ \\
\hline
\end{tabular}

O grupo gerador é o bem adquirido de maior valor econômico; sendo assim, a sua vida útil, que corresponde a 10 anos, foi utilizada para elaborar o fluxo de caixa do projeto, com taxa de desconto de 17,88\% ao ano (BNDES, 2016). Os juros obtidos para o capital investido estão representados na Tabela 6.

Tabela 6 - Juros sobre o capital de investimento

\begin{tabular}{|c|c|c|c|}
\hline $\begin{array}{c}\text { Equipamento / } \\
\text { material }\end{array}$ & $\begin{array}{c}\text { Valor inicial } \\
\text { (R\$) }\end{array}$ & $\begin{array}{c}\text { Taxa de } \\
\text { juros } \\
\text { (\%/ano) }\end{array}$ & $\begin{array}{c}\text { Juros } \\
\text { (R\$ / ano) }\end{array}$ \\
\hline $\begin{array}{c}\text { Abrigo do conjunto } \\
\text { motor-gerador }\end{array}$ & $\mathrm{R} \$ 0,00$ & $17,88 \%$ & $\mathrm{R} \$ 0,00$ \\
\hline Instalações elétricas & $\mathrm{R} \$ 1.155,00$ & $17,88 \%$ & $\mathrm{R} \$ 103,24$ \\
\hline Grupo gerador & $\mathrm{R} \$ 16.500,00$ & $17,88 \%$ & $\mathrm{R} \$ 1.474,75$ \\
\hline Total & $\mathrm{R} \$ 17.655,00$ & & $\mathrm{R} \$ 1.578,00$ \\
\hline
\end{tabular}

Não foi considerado o valor final, pois os valores de revenda não foram obtidos.

Os custos de operação e manutenção em função do tempo de uso por dia do gerador e intervalo de manutenção de cada item estão descritos na tabela 7.

Tabela 7 - Operação e manutenção do conjunto motor-gerador.

\begin{tabular}{|c|c|c|c|c|c|c|c|}
\hline \multicolumn{2}{|c|}{$\mathbf{N}^{\mathbf{0}}$ de horas de funcionamento do gerador por dia } & \multicolumn{2}{c|}{$\mathbf{1 0}$} & \multicolumn{2}{|c|}{$\mathbf{1 5}$} & $\mathbf{2 0}$ \\
\hline Componente & $\begin{array}{c}\text { Intervalo } \\
\text { (h) }\end{array}$ & $\begin{array}{c}\text { Custo de } \\
\text { O\& M (R\$) }\end{array}$ & \multicolumn{2}{c|}{ Custo anual de operação e manutenção O \& M (R\$) } \\
\hline Troca de óleo & 100 & 200,00 & $7.300,00$ & $10.950,00$ & $14.600,00$ & $17.520,00$ \\
\hline Troca de filtro & 400 & 50,00 & 456,25 & 684,38 & 912,50 & $1.095,00$ \\
\hline Limpeza dos filtros & 100 & 50,00 & $1.825,00$ & $2.737,50$ & $3.650,00$ & $4.380,00$ \\
\hline $\begin{array}{c}\text { Limpeza das } \\
\text { válvulas }\end{array}$ & 2000 & 250,00 & 456,25 & 684,38 & 912,50 & $1.095,00$ \\
\hline Troca do filtro de ar & 500 & 150,00 & $1.095,00$ & $1.642,50$ & $2.190,00$ & $2.628,00$ \\
\hline Rolamento & 12000 & 370,00 & 112,54 & 168,81 & 225,08 & 270,10 \\
\hline & Total (R\$) & & $\mathbf{1 1 . 2 4 5 , 0 4}$ & $\mathbf{1 6 . 8 6 7 , 5 6}$ & $\mathbf{2 2 . 4 9 0 , 0 8}$ & $\mathbf{2 6 . 9 8 8 , 1 0}$ \\
\hline
\end{tabular}

Fonte: Valores e estimativa para tempo de troca fornecido pelo mecânico.

A tabela 8 resume os valores de custo de depreciação, juros e manutenção preventiva em relação ao tempo de funcionamento do gerador por dia.

Tabela 8 - Custos totais de operação do sistema de cogeração

\begin{tabular}{|c|c|c|c|c|}
\hline $\begin{array}{c}\text { No de horas de funcionamento } \\
\text { do gerador por dia }\end{array}$ & $\mathbf{1 0}$ & $\mathbf{1 5}$ & $\mathbf{2 0}$ & $\mathbf{2 4}$ \\
\hline Itens de custo & $\mathbf{( R \$ / a n o )}$ & $\mathbf{( R \$ / a n o )}$ & $\mathbf{( R \$ / a n o )}$ & (R\$/ano) \\
\hline Depreciação & $1.707,75$ & $1.707,75$ & $1.707,75$ & $1.707,75$ \\
\hline Juros & $1.578,00$ & $1.578,00$ & $1.578,00$ & $1.578,00$ \\
\hline Manutenção preventiva & $11.245,04$ & $16.867,56$ & $22.490,08$ & $26.988,10$ \\
\hline Total & $\mathbf{1 4 . 5 3 0 , 8 0}$ & $\mathbf{2 0 . 1 5 3 , 3 2}$ & $\mathbf{2 5 . 7 7 5 , 8 4}$ & $\mathbf{3 0 . 2 7 3 , 8 5}$ \\
\hline
\end{tabular}


A tabela 9 resume a produção de energia, o benefício, o custo com manutenção o VLP com as variações de tempo de operação. Para cálculo do benefício foi considerada a taxa de energia elétrica de R\$0,66 kwh (ELETROBRAS, jan/2016).

Tabela 9 - Simulação da produção de energia com variação do tempo de operação

\begin{tabular}{|c|c|c|c|c|c|c|}
\hline $\begin{array}{c}\text { Produção de } \\
\text { energia } \\
\text { (KWh/ano) }\end{array}$ & Operação (h) & Benefício (R\$) & Custo (R\$) & VLP & $\begin{array}{c}\text { TIR } \\
\text { (\%) }\end{array}$ & Playback \\
\hline $\mathbf{2 3 . 7 2 5 , 0 0}$ & $\mathbf{1 0}$ & $\mathbf{1 5 . 7 5 0 , 9 8}$ & $\mathbf{1 2 . 9 5 2 , 7 9}$ & $\mathbf{1 9 3 , 4 5}$ & $\mathbf{8 , 7}$ & $\mathbf{6 , 4 9}$ \\
\hline $35.587,50$ & 15 & $23.626,47$ & $18.575,31$ & 13817,88 & 24,7 & 3,59 \\
\hline $47.450,00$ & 20 & $31.501,96$ & $24.197,83$ & 27442,32 & 38,7 & 2,48 \\
\hline $56.940,00$ & 24 & $37.802,35$ & $28.695,85$ & 38341,87 & 49,2 & 1,99 \\
\hline
\end{tabular}

\subsubsection{Cenário 02}

Converter toda a energia produzida no cenário 01 para rede de distribuição conforme resolução da Aneel, durante as 24 horas por dia, nas quais o empreendimento pode chegar a produzir 56.940 kwh por ano ou 4,7 MWh por mês, enqua- drando-se na segunda categoria de pequenos produtores de energia elétrica, a da minigeração distribuída, gerando retorno de investimento em 2 anos, com benefício de até $\mathrm{R} \$ 37.800,00$ e custo de operação e manutenção do gerador + filtros $\mathrm{R} \$ 28.700,00$, conforme tabela 10.

Tabela 10 - Simulação da produção de energia com tempo de operação de 24 horas

\begin{tabular}{|c|c|c|c|c|c|c|}
\hline $\begin{array}{c}\text { Produção de } \\
\text { energia } \\
\text { (KWh/ano) }\end{array}$ & Operação (h) & Benefício (R\$) & Custo (R\$) & VLP & $\begin{array}{c}\text { TIR } \\
\text { (\%) }\end{array}$ \\
\hline $56.940,00$ & 24 & $37.802,35$ & $28.695,85$ & 38341,87 & 49,2 & 1,99 \\
\hline
\end{tabular}

\section{DISCUSSÃO}

A estimativa da produção real de metano foi de $39,00 \mathrm{~m}^{3} \mathrm{dia}^{-1}\left(0,16 \mathrm{Nm}^{3} / \mathrm{m}^{3}\right.$ de esgoto $)$ e $(0,227$ $\left.\mathrm{Nm}^{3} \mathrm{~kg}_{\text {DoOrem }}{ }^{-1}\right)$, valor pouco abaixo do encontrado na literatura, 0,18 Nm3 $\mathrm{kg}_{\text {DQOorem }}{ }^{-1}$ Noyola, Capdeville e Roques (1988) e 0,24 $\mathrm{Nm}^{3} \mathrm{~kg}_{\text {DoOrem }}{ }^{-1}$ Eller (2013) e a estimativa do biogás capturado $60,00 \mathrm{~m}^{3}$ dia-1 $2,50 \mathrm{~m}^{3} \mathrm{~h}^{-1}\left(0,246 \mathrm{Nm}^{3} / \mathrm{m}^{3}\right.$ de esgoto) está coerente com os valores encontrados por ELLER (2013).

Para o cálculo do poder calorífico inferior disponibilizado pelo $\mathrm{CH} 4$ (PCID) foi adotado $65 \%$ de metano no biogás (Zilotti, 2012). Todavia, quanto maior a parcela de metano, maior será a capacidade calorífica do biogás, e essa proporção dependerá sobretudo das condições operacionais da estação de tratamento e das características fí- sico-químicas do esgoto afluente (COELHO et al., 2004; COSTA, 2006).

A queima da produção real de metano forneceria uma potência elétrica de $252,59 \mathrm{kWh} / \mathrm{d}$. No caso de motores de combustão interna, a eficiência de geração de energia elétrica varia numa faixa entre 33 e $36 \%$ (de acordo com levantamento realizado por Lobato, 2011), utilizando $35 \%$, o que geraria uma potência elétrica efetiva de 88,41 $\mathrm{kWh} / \mathrm{d}$ com potencial de geração de energia elétrica de 2.689,08 kwh/ mês ou 32.688,96 kwh/ ano. Em outro caso, levando em conta a potência disponível para aproveitamento em sistema de ciclo combinado (considerando o aproveitamento da energia na forma de energia elétrica e calor), a eficiência de sistemas empregando motores de combustão interna está entre 75 e 76\% (LOBATO, 
2011). Nessas condições, a queima do biogás produzido na ETE disponibilizaria uma potência efetiva total de $189,45 \mathrm{kWh} / \mathrm{d}$ para aproveitamento conjunto na geração de energia elétrica e calor com potencial de geração de energia elétrica de $5.762,31$ kwh/ mês ou $69.147,77$ kwh/ano.

Demanda de iluminação dos postes

Comprimento das ruas do condomínio: $1.090 \mathrm{~m}$

$$
N \text { o de postes }=\frac{\text { Comprimento da rua em metros }}{35}=\frac{1090}{35}=32 \text { postes }
$$

Cálculo de demanda de iluminação, considerando potência de $0,15 \mathrm{kVA}$ referente à lâmpada de $125 \mathrm{~W}$ Demanda de iluminação $=\mathrm{N}^{\circ}$ de postes $\times \mathrm{P}$ da luminária em kVA $=32 \times 0,15=4,8$ kVA

Demanda de iluminação na área de lazer Espaço gourmet - área do ambiente de $9 \mathrm{~m}^{2}$ (são 4 ambientes) - $400 \mathrm{VA}$ Apoio para espaço gourmet - área de $37 \mathrm{~m}^{2}$ - 520VA Quadra poliesportiva -4 refletores de $250 \mathrm{~W}=$ $1,16 \mathrm{kVA}$

Demanda total de iluminação do espaço gourmet $\mathrm{e}$ quadra poliesportiva: $0,40+0,52+1,16=2,06 \mathrm{kVA}$.

\subsection{Cenário 01}

Com a operação de 10 horas por dia, os benefícios anuais obtidos foram de $\mathrm{R} \$ 15.750,98$, e os custos anuais, de $\mathrm{R} \$ 12.952,79$. A data zero corresponde ao valor do investimento inicial de $R \$ 18.150,00$. De acordo com esses dados, os resultados mostraram que o investimento é viável economicamente quando o período de utilização for de 10 horas por dia, quando a produção atingir 23.725,00 KWh/ ano, com valor de VLP de R $\$ 193,45$, TIR $8,76 \%$ e PRC de 6,49 anos.

O condomínio produz $2,5 \mathrm{~m}^{3}$ de biogás por hora com população de 2.544 habitantes. Para o condomínio gerar pelo menos $4 \mathrm{~m}^{3}$ por hora, seria necessária uma população de pelo menos 4.070 habitantes para uma estação com as mesmas características. Para melhorar essa produção, pode-se melhorar a eficiência da remoção do DQQ da estação de tratamento, considerando que a estação atinja $75 \%$ de eficiência de remoção, são produzidos $2,83 \mathrm{~m}^{3}$ de biogás por hora; portanto, quanto maior a eficiência de remoção, melhor é o aproveitamento do biogás para produção de energia.

\subsection{Cenário 02}

O benefício gerado é decorrente da energia retornada para rede, que entra como compensação de crédito da próxima fatura, ou seja, não é um valor que o empreendimento vai receber, mas um valor que o empreendimento vai deixar de pagar.

\section{CONCLUSÃO}

A utilização do biogás não deve ser tratada apenas como um interesse econômico. Ao utilizá-lo como fonte de energia elétrica nos motores de combustão ou apenas queimá-lo, evita-se a emissão de gás metano, que possui maior potencial de poluição comparado ao dióxido de carbono, para a atmosfera.

Baseado na população do empreendimento onde foi feito o estudo, conclui-se que o potencial elétrico gerado por habitante pode chegar a 0,07 $\mathrm{kWh} / \mathrm{d}$ em função da tecnologia de geração utilizada, sendo capaz de alcançar a capacidade anual de até 27,18 kwh/ano. 
Em função dos dois cenários, observa-se que:

No primeiro cenário, a geração de energia promovida pelo gerador de $18 \mathrm{cv}$ é suficiente para atender à iluminação da área de lazer e das ruas internas por meio dos postes.

A simulação mostrou que a partir de 10 horas de operação o investimento é viável. No entanto, a população do condomínio não é suficiente para gerar $4 \mathrm{~m}^{3}$ de biogás por hora para dar partida ao gerador, portanto seria necessário outro equipamento com melhor eficiência.

Já no cenário 2 observa-se que a implementação no empreendimento da Resolução da ANEEL 687/15 mostrou-se bastante viável, visto que toda a energia gerada pode ir para a rede de distribuição, sem gastos a mais, como baterias acumuladoras de energia ou reservatórios para acúmulo de biogás, onde o sistema apresentou grande vantagem para o empreendimento, visto que necessita apenas de 2 anos para retorno do capital investido.

Apesar das vantagens da microgeração em nível de eficiência energética e preservação do ambiente, o elevado custo do sistema, a falta de incentivo público e a baixa eficiência de geração dificultam a sua implementação.

É essencial a continuação de pesquisas nessa área e o incentivo às empresas para que sejam implantados projetos e estudos desse tipo.

\section{REFERÊNCIAS BIBLIOGRÁFICAS}

AGÊNCIA NACIONAL DE ENERGIA ELÉTRICA - ANEEL. Guia do cogerador de energia elétrica. Brasília: Distrito Federal, 1998. (Série Estudos e Informações Hidrológicas e Energéticas, 5).

AGROSHOP- disponível em http://www.agroshop.com.br/geradorbiogas. Acessado em 19 de novembro de 2015.

ANEEL. Resolução Normativa n 687, de 24 de novembro de 2015, referências disponíveis na Internet, http://www2.aneel.gov.br/cedoc/ren2015687.pdf, 2015.
ARCADIS TETRAPLAN. Estudo sobre o Potencial de Geração de Energia a partir de Resíduos de Saneamento (lixo, esgoto), visando incrementar o uso de biogás como fonte alternativa de energia renovável. São Paulo. 2010.

ASSOCIAÇÃO BRASILEIRA DE NORMAS TÉCNICAS, NBR 5410: Instalações elétricas de baixa tensão, 2005.

BARBIRATO, G. M.; BARBOSA, R. V. R.; FERNANDES, E. F.; MORAIS, C. S.- Análise de Perfis Térmicos Urbanos em Maceió - AL; In: IX Encontro Nacional de Tecnologia do Ambiente Construído, 2002.

BAUER, U. R. Matemática Financeira Fundamental. São Paulo: Atlas, 2008.

BILOTTA. P. Determinação da Capacidade de Geração de Energia da Estação de Tratamento de Esgotos Santa Quitéria em Curitiba/PR a Partir do Biogás. Especialização em Projetos Sustentáveis, Mudanças Climáticas e Gestão Corporativa de Carbono do Programa de Educação Continuada em Ciências Agrárias, da Universidade Federal do Paraná. 21p. 2014.

BNDES - Banco Nacional do Desenvolvimento - taxa de juros, 2016. Disponível em https://www.cartaobndes.gov.br/cartaobndes/PaginasCartao/Taxa.asp?Acao=L Acesso em 15 de fevereiro de 2016.

COSTA, D. F. Geração de energia elétrica a partir do biogás de tratamento de esgoto. Dissertação (Mestrado em Energia) - Programa Inter-unidades de Pós-Graduação em Energia, Universidade de São Paulo, São Paulo-SP, 194p. 2006.

CROOK, J. Applications, risks, and public acceptance of water reuse. Apresentação oral. Trabalho apresentado no $1^{\circ}$ Simpósio Internacional de Reúso de Água, ABES-PR, Curitiba, 2012.

ELETROBRAS - Plano Nacional de Energia Elétrica 1993-2015. Estudos Básicos Volume III Projeto 4. A oferta de Energia Elétrica. Biomassa Floresta, 1994

FIBRATÉCNICA - Projeto básico e memorial descritivo da estação de tratamento PARK SHOPPING CONDOMÍNIO CLUBE- 2011

FLORENCIO, L.; BASTOS, R.; AISSE, M. M. Tratamento e utilização de esgotos sanitários. 1.ed. Rio de Janeiro.RJ: RiMa/ABES - Projeto PROSAB, 2006. Fn Spon, 1997. 526 p.

HELMER, R.; HESPANHOL, I. Water pollution control - a guide to the use of water quality management principles. Londres: $\mathrm{Pu}$ blished on Behalf of Who by F \& Fn Spon, 1997. 526 p.

HOLM-NIELSEN, J.B.; AL SEADI, T.; OLESKOWICZ-POPIEL, P. The future of anaerobic digestion and biogas utilization. Bioresource Technology, v. 100, p. 5478-5484. 2009.

JIMÉNEZ, B. ASANO, T. Water reuse: an international survey of current practice, issues and needs. 1.ed. Londres: IWA Publishing, 2008. 
Lapponi, J. C., Projetos de Investimento: Construção e Avaliação de Fluxo de Caixa: Modelos em Excel. Lapponi Treinamento e Editora, São Paulo, 2000.

LIBHABER, M. Experiência de reúso de água em Israel: apresentação oral. Trabalho apresentado no $1^{\circ}$ Simpósio Internacional de Reúso de Água Simpósio, ABES-PR, Curitiba, 2012.

LOBATO, L. C. S. Aproveitamento energético de biogás gerado em reatores UASB tratando esgoto doméstico. $187 \mathrm{f}$. Tese (Doutorado em Saneamento, Meio Ambiente e Recursos Hídricos) - Universidade Federal de Minas Gerais, Belo Horizonte, MG, 2011.

NOYOLA, A.; CAPDEVILLE, B.; ROQUUES, H. Anaerobic treatment of domestic sewage with a rotating stationary fixed-film reactor. Water Research, v. 22, n. 12, p. 1585-1592. 1988.

NORMA TÉCNICA - NTD - 001/2008: Projeto de Loteamentos Departamento de Projeto e Construção
POLONIO, J. C. et al. Potencial biorremediador de microrganismos: Levantamento de resíduos industriais e urbanos tratáveis no município de Maringá-PR. BBR - BIOCHEMISTRY AND BIOTECHNOLOGY REPORTS, Londrina, v.3, n.2, p.31-45, 2014. E-ISSN: 2316-5200

VON SPERLING, M. Princípios básicos do tratamento de esgoto. 3. ed. Belo Horizonte: Departamento de Engenharia Sanitária e Ambiental. Universidade Federal de Minas Gerais, 1996. 211 p. (Princípios do tratamento biológico de águas residuárias, v.2).

WORLD POPULATION; United Nations Department of Economic and Social Affairs Population Division. 15-15398—Dezembro 2015

ZILOTTI, H. A. R. Potencial de Produção de Biogás em uma Estação de Tratamento de Esgoto de Cascavel para a Geração de Energia Elétrica. Programa de Pós-Graduação Stricto Sensu em Energia na Agricultura. Universidade Estadual do Oeste do Paraná- UNIOESTE, Paraná. 52p. 2012. 